\title{
Solution of Several Functional Equations on Nonunital Semigroups Using Wilson's Functional Equations with Involution
}

\author{
Jaeyoung Chung ${ }^{1}$ and Prasanna K. Sahoo ${ }^{2}$ \\ ${ }^{1}$ Department of Mathematics, Kunsan National University, Gunsan 573-701, Republic of Korea \\ ${ }^{2}$ Department of Mathematics, University of Louisville, Louisville, KY 40292, USA \\ Correspondence should be addressed to Jaeyoung Chung; jychung@kunsan.ac.kr
}

Received 23 April 2014; Revised 29 July 2014; Accepted 6 August 2014; Published 27 August 2014

Academic Editor: Alberto Fiorenza

Copyright ( $) 2014$ J. Chung and P. K. Sahoo. This is an open access article distributed under the Creative Commons Attribution License, which permits unrestricted use, distribution, and reproduction in any medium, provided the original work is properly cited.

Let $S$ be a nonunital commutative semigroup, $\sigma: S \rightarrow S$ an involution, and $\mathbb{C}$ the set of complex numbers. In this paper, first we determine the general solutions $f, g: S \rightarrow \mathbb{C}$ of Wilson's generalizations of d'Alembert's functional equations $f(x+y)+$ $f(x+\sigma y)=2 f(x) g(y)$ and $f(x+y)+f(x+\sigma y)=2 g(x) f(y)$ on nonunital commutative semigroups, and then using the solutions of these equations we solve a number of other functional equations on more general domains.

\section{Introduction}

The functional equation

$$
f(x+y)+f(x-y)=2 f(x) f(y)
$$

is known as the d'Alembert's functional equation. It has a long history going back to d'Alembert [1]. As the name suggests this functional equation was introduced by d'Alembert in connection with the composition of forces and plays a central role in determining the sum of two vectors in Euclidean and non-Euclidean geometries [2].

Several authors have determined the general solution $f$ : $S \rightarrow \mathbb{F}$ of the functional equation

$$
f(x+y)+f(x+\sigma y)=2 f(x) f(y),
$$

for all $x, y \in S$, where $\sigma: S \rightarrow S$ is an automorphism of order 2. Stetkær [3] studied (2) when $\mathbb{F}=\mathbb{C}, S$ is an abelian topological group and $f: S \rightarrow \mathbb{C}$ and $\sigma: S \rightarrow S$ are continuous. Sinopoulos [4] determined the general solution $f: S \rightarrow \mathbb{F}$ of (2) when $S$ is a commutative semigroup, $\mathbb{F}$ is a quadratically closed commutative field of characteristic different from 2, and $\sigma$ is an endomorphism of $S$ of order 2.

Wilson's functional equation $f(x+y)+f(x-y)=$ $2 f(x) g(y)$ is a generalization of d'Alembert's functional equation. Among others Wilson's functional equation was studied by Wilson [5] and [6], Kaczmarz [7], van der Lyn [8], Fenyö [9], Chung et al. [10], and Aczél [11]. Stetkær (see [12] and [3]) studied Wilson's equation with an involution; namely,

$$
f(x+y)+f(x+\sigma y)=2 f(x) g(y), \quad \forall x, y \in S,
$$

where $S$ is a topological monoid (i.e., $S$ is a semigroup with an identity element), $f, g$ are continuous functions defined on $S$ and taking values on $\mathbb{C}$, and $\sigma: S \rightarrow S$ is a continuous automorphism of order 2 . He gave several properties of the solution $(f, g)$ of Wilson's equation (see [3, Chapter 11, Lemmas 11.3 and 11.4]). In Chapter 11 of [3] (see Corollary 11.7) a complete and satisfactory description of the solutions of Wilson's equation (3) was given on abelian groups.

The functional equation $f(x+y)+f(x-y)=2 g(x) f(y)$ was introduced in [6] (see also [3] Chapter 11). For more on known results related to Wilson's functional equations we refer the reader to [13-20]. The above functional equation with an involution, namely,

$$
f(x+y)+f(x+\sigma y)=2 g(x) f(y), \quad \forall x, y \in S,
$$

was studied by Stetkær (see [21]) on abelian groups. 
In this paper, first we present the solutions of Wilson's functional equations (3) and (4) on nonunital commutative semigroups and then using the general solutions of these functional equations we determine the general solutions of several functional equations in two variables in more general domains. In particular, we determine the general solutions $(f, g)$ of functional equations; namely,

$$
\begin{array}{r}
f(p r, q s)+f(p s, q r)=2 f(p, q) g(r, s) \\
\forall p, q, r, s \in(0,1], \\
f(p r, q s)+f(p s, q r)=2 g(p, q) f(r, s) \\
\forall p, q, r, s \in(0,1]
\end{array}
$$

treated by Riedel and Sahoo in [22], on more general domains. We also determine the general solutions of functional equations:

$$
\begin{gathered}
f(z w)+f(z \bar{w})=2 f(z) g(w) \quad \forall z, w \in \mathbb{C}, \\
f(z w)+f(z \bar{w})=2 g(z) f(w) \quad \forall z, w \in \mathbb{C}, \\
f(u x-v y, u y+v x)+f(u x+v y, u y-v x) \\
=2 f(x, y) g(u, v) \quad \forall x, y, u, v \in \mathbb{R}, \\
f(u x+v y, u y+v x)+f(u x-v y, u y-v x) \\
\quad=2 f(x, y) g(u, v) \quad \forall x, y, u, v \in \mathbb{R} .
\end{gathered}
$$

\section{Solution of Wilson's Equations on Commutative Nonunital Semigroups}

In this section, we present the solutions $(f, g)$ of Wilson's functional equations (3) and (4) on nonunital semigroups. We solve these equations on more general domains than the domains used in [3]. In particular we give the description of the solutions of Wilson's equations (3) and (4) on nonunital semigroups whereas in [3] the description of the solutions of (3) is given on commutative topological monoids (i.e., semigroups with an identity element). Note that Lemmas 11.3 and 11.4 of Chapter 11 in [3] are not very useful for solving (3) and (4) on nonunital commutative semigroups due to the lack of identity element. Our method of proof is quite elementary and transparent.

Throughout this paper we denote by $S$ a 2 -divisible commutative semigroup with no identity element and denote by $\mathbb{C}$ the set of complex numbers. A function $\sigma: S \rightarrow S$ is said to be an involution if $\sigma(x+y)=\sigma(y)+\sigma(x)$, for all $x, y \in S$, and $\sigma(\sigma(x))=x$, for all $x \in S$. For simplicity we write $\sigma x$ instead of $\sigma(x)$. A function $m: S \rightarrow \mathbb{C}$ is called an exponential function provided that $m(x+y)=m(x) m(y)$, for all $x, y \in S$, and $a: S \rightarrow \mathbb{C}$ is called an additive function provided that $a(x+y)=a(x)+a(y)$, for all $x, y \in S$.

As a direct consequence of a theorem of Sinopoulos [4] we have the following lemma.

Lemma 1. Let $g: S \rightarrow \mathbb{C}$ satisfy

$$
g(x+y)+g(x+\sigma y)=2 g(x) g(y)
$$

for all $x, y \in S$. Then there exists an exponential function $m$ : $S \rightarrow \mathbb{C}$ such that

$$
g(x)=\frac{m(x)+m(\sigma x)}{2}
$$

for all $x \in S$.

In the following, we exclude the trivial cases when $f(x) \equiv$ 0 or $g(x) \equiv 0$.

Theorem 2. Let $f, g: S \rightarrow \mathbb{C}$ satisfy the functional equation

$$
f(x+y)+f(x+\sigma y)=2 f(x) g(y)
$$

for all $x, y \in S$. Then either $g, f$ are of the form

$$
g(x)=\frac{m(x)+m(\sigma x)}{2}, \quad f(x)=\alpha_{1} m(x)+\alpha_{2} m(\sigma x),
$$

for all $x \in S$, where $m: S \rightarrow \mathbb{C}$ is an exponential function satisfying $m \neq m \circ \sigma$ and $\alpha_{1}, \alpha_{2} \in \mathbb{C}$, or else

$$
f(x)= \begin{cases}m(x)(\beta+a(x)), & \text { if } x \in S \backslash K, \\ 0 & \text { if } x \in K,\end{cases}
$$

where $m: S \rightarrow \mathbb{C}$ is an exponential function satisfying $m=$ $m \circ \sigma, K=\{x \in S \mid m(x)=0\}$ and $a: S \backslash K \rightarrow \mathbb{C}$ is an additive function satisfying $a=-a \circ \sigma$ and $\beta \in \mathbb{C}$.

Proof. Choose an $x_{0} \in S$ such that $f\left(x_{0}\right) \neq 0$. Replacing $x$ by $x_{0}$ in (9) and dividing the result by $2 f\left(x_{0}\right)$ we have

$$
g(y)=\frac{f\left(x_{0}+y\right)+f\left(x_{0}+\sigma y\right)}{2 f\left(x_{0}\right)}
$$

for all $y \in S$. Multiplying both sides of (12) by $2 g(x)$ and using (9) we have

$$
\begin{aligned}
2 g(y) g(x) & \frac{2 f\left(x_{0}+y\right) g(x)+2 f\left(x_{0}+\sigma y\right) g(x)}{2 f\left(x_{0}\right)} \\
= & \left(f\left(x_{0}+y+x\right)+f\left(x_{0}+y+\sigma x\right)\right. \\
& \left.\quad+f\left(x_{0}+\sigma y+x\right)+f\left(x_{0}+\sigma y+\sigma x\right)\right) \times\left(2 f\left(x_{0}\right)\right)^{-1} \\
= & \frac{f\left(x_{0}+y+x\right)+f\left(x_{0}+\sigma y+\sigma x\right)}{2 f\left(x_{0}\right)} \\
& +\frac{f\left(x_{0}+y+\sigma x\right)+f\left(x_{0}+\sigma y+x\right)}{2 f\left(x_{0}\right)} \\
= & \frac{f\left(x_{0}+y+x\right)+f\left(x_{0}+\sigma(y+x)\right)}{2 f\left(x_{0}\right)} \\
& +\frac{f\left(x_{0}+y+\sigma x\right)+f\left(x_{0}+\sigma(y+\sigma x)\right)}{2 f\left(x_{0}\right)} \\
g & (y+x)+g(y+\sigma x)
\end{aligned}
$$


for all $x, y \in S$. By Lemma $1, g$ has the form

$$
g(x)=\frac{m(x)+m(\sigma x)}{2}
$$

for all $x \in S$. Now, we find $f$. Let $f_{e}$ and $f_{o}$ be the even part and the odd part of $f$ with respect to $\sigma$; that is,

$$
\begin{aligned}
& f_{e}(x)=\frac{1}{2}(f(x)+f(\sigma x)), \\
& f_{o}(x)=\frac{1}{2}(f(x)-f(\sigma x))
\end{aligned}
$$

for all $x, y \in S$. Then we have

$$
\begin{aligned}
& f(x)=f_{e}(x)+f_{o}(x), \quad f_{e}(\sigma x)=f_{e}(x), \\
& f_{o}(\sigma x)=-f_{o}(x)
\end{aligned}
$$

for all $x \in S$. Replacing $x$ by $\sigma x$ in (14) we obtain

$$
g(\sigma x)=g(x)
$$

for all $x \in S$. Replacing $(x, y)$ by $(\sigma x, \sigma y)$ in (9) and using (17) we have

$$
f(\sigma x+\sigma y)+f(\sigma x+y)=2 f(\sigma x) g(y)
$$

for all $x, y \in S$. Adding (18) to (9) and dividing the result by 2 we have

$$
f_{e}(x+y)+f_{e}(x+\sigma y)=2 f_{e}(x) g(y)
$$

for all $x, y \in S$. Replacing $(x, y)$ by $(y, x)$ in (19) and using (16) we have

$$
f_{e}(x+y)+f_{e}(x+\sigma y)=2 f_{e}(y) g(x) .
$$

Putting $y=y_{0}$ in (19) and (20) such that $g\left(y_{0}\right) \neq 0$ and equating the right hand sides of the results we have

$$
f_{e}(x)=\frac{f_{e}\left(y_{0}\right)}{g\left(y_{0}\right)} g(x):=\beta g(x)
$$

for all $x \in S$. From now on, we determine $f_{o}$. Subtracting (18) from (9) and dividing the result by 2 we have

$$
f_{o}(x+y)+f_{o}(x+\sigma y)=2 f_{o}(x) g(y)
$$

for all $x, y \in S$. Replacing $(x, y)$ by $(y, x)$ and using $(16)$ we have

$$
f_{o}(x+y)-f_{o}(x+\sigma y)=2 f_{o}(y) g(x)
$$

for all $x, y \in S$. Subtracting (23) from (22) and dividing the result by 2 we have

$$
f_{o}(x+\sigma y)=f_{o}(x) g(y)-f_{o}(y) g(x)
$$

for all $x, y \in S$. We divide into two cases when (i) $m(\sigma x) \neq$ $m(x)$, for some $x \in S$, and (ii) $m(\sigma x)=m(x)$, for all $x \in S$.

Case 1. First, we consider the case when $m(\sigma x) \neq m(x)$, for some $x \in S$. We show that if $m \neq m \circ \sigma$, then $g$ is not an exponential function. Indeed, from (10) we have

$$
\begin{aligned}
g(x+y) & =\frac{1}{2}(m(x+y)+m(\sigma x+\sigma y)) \\
& =\frac{1}{2}(m(x) m(y)+m(\sigma x) m(\sigma y))
\end{aligned}
$$

for all $x, y \in S$, and

$$
g(x) g(y)=\frac{1}{4}(m(x)+m(\sigma x))(m(y)+m(\sigma y))
$$

for all $x, y \in S$. If $g$ is exponential, equating (25) and (26) and multiplying the result by 4 we have

$$
(m(x)-m(\sigma x))(m(y)-m(\sigma y))=0
$$

for all $x, y \in S$, which implies $m(x)=m(\sigma x)$, for all $x \in S$, contradicting our assumption that $m(x) \neq m(\sigma x)$, for some $x \in S$. Thus, $g$ is not exponential. Let

$$
f_{o_{p}}(x)=\frac{1}{2}(m(x)-m(\sigma x)) .
$$

Then it is easy to see that $\left(f_{p}, g\right)$ satisfies the functional equation (22). Since $g$ is not exponential, we can choose $x_{0}, y_{0} \in S$ such that $g\left(x_{0}+y_{0}\right) \neq g\left(x_{0}\right) g\left(y_{0}\right)$. Let $f_{o}$ be an arbitrary solution of the functional equation (22). We can choose $c_{1}, c_{2} \in \mathbb{C}$ (not both zero) such that

$$
c_{1} f_{o}\left(x_{0}\right)+c_{2} f_{o_{p}}\left(x_{0}\right)=0 .
$$

Let

$$
h_{o}(x)=c_{1} f_{o}(x)+c_{2} f_{o_{p}}(x)
$$

for all $x \in S$. Then $h_{0}\left(x_{0}\right)=0$ and $h_{o}$ satisfies the functional equation (22) and satisfies

$$
h_{o}(x+\sigma y)=h_{o}(x) g(y)-h_{o}(y) g(x)
$$

for all $x, y \in S$. We claim that $h_{0}(x) \equiv 0$. If not, that is, there exists $\alpha \in S$ such that $h_{0}(\alpha) \neq 0$; then putting $y=\alpha$ in (31) and dividing the result by $h_{0}(\alpha)$ we have

$$
g(x)=\frac{h_{o}(x) g(\alpha)-h_{o}(x+\sigma \alpha)}{h_{o}(\alpha)}
$$

for all $x \in S$. Putting $y=\sigma x_{0}$ in (31) and using (17) and (30) we have

$$
\begin{aligned}
h_{o}\left(x+x_{0}\right) & =h_{o}(x) g\left(\sigma x_{0}\right)-h_{0}\left(\sigma x_{0}\right) g(x) \\
& =h_{o}(x) g\left(x_{0}\right)+h_{0}\left(x_{0}\right) g(x) \\
& =h_{o}(x) g\left(x_{0}\right)
\end{aligned}
$$

for all $x \in S$. Now, from (32) and (33) we have

$$
\begin{aligned}
g\left(x_{0}\right) g(x)= & \frac{-h_{o}\left(x_{0}+\sigma \alpha\right)\left(h_{o}(x) g(\alpha)-h_{o}(x+\sigma \alpha)\right)}{h_{o}(\alpha)^{2}} \\
= & \left(h_{o}(\alpha) g\left(x_{0}\right) h_{0}(x) g(\alpha)\right. \\
& \left.\quad-h_{o}(\alpha) g\left(x_{0}\right) h_{o}(x+\sigma \alpha)\right) \times\left(h_{o}(\alpha)^{2}\right)^{-1} \\
= & \frac{g\left(x_{0}\right) h_{0}(x) g(\alpha)-g\left(x_{0}\right) h_{o}(x+\sigma \alpha)}{h_{o}(\alpha)} \\
= & \frac{h_{0}\left(x+x_{0}\right) g(\alpha)-h_{o}\left(x+x_{0}+\sigma \alpha\right)}{h_{o}(\alpha)} \\
= & g\left(x+x_{0}\right) \quad
\end{aligned}
$$


for all $x \in S$, which contradicts the fact that $g\left(x_{0}+y_{0}\right) \neq$ $g\left(x_{0}\right) g\left(y_{0}\right)$. Thus, we conclude that $h_{0}(x) \equiv 0$ and it follows from (30) that

$$
c_{1} f_{o}(x)+c_{2} f_{o_{p}}(x)=0
$$

for all $x \in S$. If $c_{1}=0$, then $c_{2} \neq 0$ and hence

$$
f_{o_{p}}(x)=\frac{1}{2}(m(x)-m(\sigma x))=0
$$

for all $x \in S$, which contracts the assumption $m(x) \neq m(\sigma x)$ for some $x \in S$. Thus, $c_{1} \neq 0$ and from (35) we have

$$
f_{o}(x)=-\frac{c_{2}}{c_{1}} f_{o_{p}}(x)
$$

for all $x \in S$. From (21), (28), and (37) we have

$$
\begin{aligned}
f(x) & =f_{e}(x)+f_{o}(x) \\
& =\beta g(x)-\frac{c_{2}}{c_{1}} f_{o_{p}}(x) \\
& =\alpha_{1} m(x)+\alpha_{2} m(\sigma x)
\end{aligned}
$$

for all $x \in S$, where $\alpha_{1}=(1 / 2)\left(\beta-\left(c_{2} / c_{1}\right)\right), \alpha_{2}=(1 / 2)(\beta+$ $\left.\left(c_{2} / c_{1}\right)\right)$. Thus, we get (10).

Case 2. Next, we assume that $m(\sigma x)=m(x)$, for all $x \in S$. Then from (14) we have

$$
g(x)=m(x), \quad m(\sigma x)=m(x)
$$

for all $x \in S$. Thus, from (24) and (39) we have

$$
f_{o}(x+\sigma y)=f_{o}(x) m(y)-f_{o}(y) m(x)
$$

for all $x, y \in S$.

Let $K=\{x \in S \mid m(x)=0\}$. Then $K$ is an ideal in $S$ and $S \backslash K$ is a subsemigroup of $S$. Dividing (40) by $m(x+\sigma y)$ and using (39) we have

$$
\frac{f_{o}(x+\sigma y)}{m(x+\sigma y)}=\frac{f_{o}(x)}{m(x)}-\frac{f_{o}(y)}{m(y)}
$$

for all $x, y \in S \backslash K$. Let

$$
a(x)=\frac{f_{o}(x)}{m(x)}
$$

for all $x \in S \backslash K$. Then we have

$$
a(x+\sigma y)=a(x)-a(y)
$$

for all $x, y \in S \backslash K$. Replacing $x$ by $y$ in (43) we have

$$
a(y+\sigma y)=a(y)-a(y)=0 .
$$

Replacing $x$ by $x+y$ in (43) we have

$$
a(x+y+\sigma y)=a(x+y)-a(y),
$$

and replacing $y$ by $y+\sigma y$ in (43) and using (44) we have

$$
a(x+y+\sigma y)=a(x)-a(y+\sigma y)=a(x) .
$$

From (45) and (46) we have

$$
a(x+y)=a(x)+a(y)
$$

for all $x, y \in S \backslash K$. Replacing $y$ by $\sigma y$ in (47) and using (43) we have

$$
a(\sigma y)=-a(y)
$$

for all $y \in S \backslash K$. From (21), (39), (42), and (48) we have

$$
\begin{aligned}
f(x) & =f_{e}(x)+f_{o}(x)=\beta g(x)+a(x) m(x) \\
& =\beta m(x)+a(x) m(x)=m(x)(\beta+a(x))
\end{aligned}
$$

for all $x \in S \backslash K$. Let $x \in K$. Then we have $m(x / 2)=$ $m(\sigma(x / 2))=0$. Thus, replacing both $x$ and $\sigma(y)$ by $(x / 2)$ in (40) we have $f_{o}(x)=0$ and also by (21) $f_{e}(x)=0$. Hence $f(x)=0$ for any $x \in K$. Thus, from (39), (48), and (49) we get (11). This completes the proof of the theorem.

Remark 3. The solution $(g, f)$ in (11) can be written in the form

$$
\begin{gathered}
g(x)=m(x+\sigma x), \\
\qquad \begin{array}{ll}
m(x+\sigma x)(\beta+a(x)-a(\sigma x)), & \text { if } x \in S \backslash K, \\
0, & \text { if } x \in K,
\end{array}
\end{gathered}
$$

where $m$ is an exponential function and $a$ is an additive function. Also, let us assume that $S$ has the property: for any $x, y \in S$, there exists a positive integer $k$ and $z \in S$ such that

$$
x+z=k y,
$$

which is satisfied by every group and also most of well known semigroups such as $S=(0,1),(0, \infty)$. Then, if $m\left(x_{0}\right)=0$ for some $x_{0} \in S$, then for any $y \in S$ we can choose a positive integer $k$ and $z \in S$ such that $x_{0}+z=k y$. Thus, we have

$$
m(y)^{k}=m(k y)=m\left(x_{0}+z\right)=m\left(x_{0}\right) m(z)=0,
$$

which implies $m(y)=0$ for all $y \in S$. Thus, $K=\{x \in S \mid$ $m(x)=0\}=\emptyset$ and $g, f$ in (11) are given by

$$
\begin{aligned}
& g(x)=m(x+\sigma x), \\
& f(x)=m(x+\sigma x)(\beta+a(x)-a(\sigma x))
\end{aligned}
$$

for all $x \in S$.

In view of the proof of Theorem 2, we can see that 2divisibility of $S$ is necessary only in showing that $f(x)=0$, for all $x \in K$. Thus, if $S$ is a commutative group possibly without 2-divisibility, we have the following. 
Corollary 4. Let $G$ be a commutative group and $f, g: G \rightarrow$ $\mathbb{C}$ satisfy the functional equation

$$
f(x+y)+f(x+\sigma y)=2 f(x) g(y)
$$

for all $x, y \in G$. Then either $g, f$ are of the form

$$
\begin{aligned}
& g(x)=\frac{m(x)+m(\sigma x)}{2}, \\
& f(x)=\alpha_{1} m(x)+\alpha_{2} m(\sigma x)
\end{aligned}
$$

for all $x \in G$, where $m$ is an exponential function satisfying $m \neq m \circ \sigma$ and $\alpha_{1}, \alpha_{1} \in \mathbb{C}$, or else

$$
g(x)=m(x), \quad f(x)=m(x)(\beta+a(x))
$$

for all $x \in G$, where $m: G \rightarrow \mathbb{C}$ is an exponential function satisfying $m=m \circ \sigma, a: G \rightarrow \mathbb{C}$ is an additive function satisfying $a=-a \circ \sigma$, and $\beta \in \mathbb{C}$.

Remark 5. Note that in general 2-divisible commutative semigroup, the solution $(g, f)$ can not be written in the simple form as above (e.g., $S=(\mathbb{R}, \times)$ : see Theorem 18$)$.

Example 6. Let $G=\left\langle\mathbb{R}^{2},+\right\rangle$ in Corollary 4 and let $a, b \in \mathbb{R}$ with $b \neq 0$ and let

$$
\sigma=\left(\begin{array}{cc}
a & b \\
\frac{1-a^{2}}{b} & -a
\end{array}\right) .
$$

Then $\sigma$ is an involution on $\mathbb{R}^{2}$ and we can consider the functional equation

$$
\begin{aligned}
& f(t+u, s+v)+f\left(t+a u+b v, s+\frac{1}{b}\left(1-a^{2}\right) u-a v\right) \\
& =2 f(t, s) g(u, v)
\end{aligned}
$$

for all $t, s, u, v \in \mathbb{R}$. As a direct consequence of Corollary 4 we can exhibit all regular solutions of (58) in a transparent form. By Corollary 4, $g$ has the form

$$
\begin{aligned}
g(t, s) & =\frac{m(t, s)+(m \circ \sigma)(t, s)}{2} \\
& =\frac{m(t, s)+m\left(a t+b s,(1 / b)\left(1-a^{2}\right) t-a s\right)}{2}
\end{aligned}
$$

for all $t, s \in \mathbb{R}$, where $m: \mathbb{R}^{2} \rightarrow \mathbb{C}$ is an exponential function. In view of the proof in [4], $m$ is given by $m(x)=$ $g(x)-\alpha\left(g\left(x+x_{0}\right)-g\left(x+\sigma x_{0}\right)\right)$, for all $x \in \mathbb{R}^{2}$ and for some $\alpha \in \mathbb{C}, x_{0} \in \mathbb{R}^{2}$. Thus, if $g$ is Lebesgue measurable, so is $m$ and has the form $m(t, s)=e^{c_{1} t+c_{2} s}$ for some $c_{1}, c_{2} \in \mathbb{C}$. Thus, $g$ has the form

$$
\begin{aligned}
& g(t, s) \\
& \quad=\frac{1}{2}\left(e^{c_{1} t+c_{2} s}+e^{(1 / b)\left(a b c_{1}+c_{2}-a^{2} c_{2}\right) t+\left(b c_{1}-a c_{2}\right) s}\right)
\end{aligned}
$$

for all $t, s \in \mathbb{R}$. Also, by Corollary 4 , if $m \neq m \circ \sigma$, that is, $c_{1} \neq(1 / b)(1+a) c_{2}$, then $f$ has the form

$$
\begin{aligned}
f(t, s)= & \alpha_{1} e^{c_{1} t+c_{2} s} \\
& +\alpha_{2} e^{(1 / b)\left(a b c_{1}+c_{2}-a^{2} c_{2}\right) t+\left(b c_{1}-a c_{2}\right) s}
\end{aligned}
$$

for all $t, s \in \mathbb{R}$ and for some $\alpha_{1}, \alpha_{2} \in \mathbb{C}$, and if $c_{1}=(1 / b)(1+$ a) $c_{2}$, then $f$ has the form

$$
f(t, s)=e^{d_{1}((1+a) t+b s)}\left(\beta+d_{2}((1-a) t+b s)\right)
$$

for all $t, s \in \mathbb{R}$ and for some $d_{1}, d_{2}, \beta \in \mathbb{C}$.

In the following theorem, we present the general solution of the second Wilson's functional equation.

Theorem 7. Let $f, g: S \rightarrow \mathbb{C}$ satisfy

$$
f(x+y)+f(x+\sigma(y))=2 g(x) f(y)
$$

for all $x, y \in S$. Then there exists an exponential function $m$ and $\beta \in \mathbb{C}$ with $\beta \neq 0$ such that

$$
f(x)=\frac{m(x)+m(\sigma x)}{2 \beta}, \quad g(x)=\frac{m(x)+m(\sigma x)}{2}
$$

for all $x \in S$.

Proof. Replacing $y$ by $\sigma y$ in (63) we see that

$$
f(y)=f(\sigma y)
$$

for all $y \in S$. Replacing $(x, y)$ by $(y, x)$ in $(63)$ and using (65) we have

$$
f(x+y)+f(x+\sigma y)=2 g(y) f(x)
$$

for all $x, y \in S$. Choosing $y_{0} \in S$ such that $g\left(y_{0}\right) \neq 0$, putting $y=y_{0}$ in (63) and (66), and equating the right hand sides of the results we have

$$
f(x)=\frac{f\left(y_{0}\right)}{g\left(y_{0}\right)} g(x):=\frac{1}{\beta} g(x)
$$

for all $x \in S$. Replacing $f(x)$ by $(1 / \beta) g(x)$ in (63) and multiplying the result by $\beta$ we have

$$
g(x+y)+g(x+\sigma y)=2 g(x) g(y)
$$

for all $x, y \in S$. By Lemma 1 and (67) we get (64). This completes the proof.

\section{Applications of Wilson's Equations in Solving Several Other Equations}

Let $(H, \circ)$ be a commutative semigroup, and $f, g: H \times H \rightarrow$ $\mathbb{C}$. As an application of Theorems 2 and 7, we determine all general solutions of the functional equations

$$
\begin{aligned}
& f(p \circ r, q \circ s)+f(p \circ s, q \circ r)=2 f(p, q) g(r, s), \\
& f(p \circ r, q \circ s)+f(p \circ s, q \circ r)=2 g(p, q) f(r, s)
\end{aligned}
$$

for all $p, q \in H$. For the case $H=(0,1],(69)$ was treated by Riedel and Sahoo [22]. In the following we exclude the trivial cases when $f=0$ or $g=0$. 
Theorem 8 (c.f. [22]). Let $f, g: H \times H \rightarrow \mathbb{C}$ satisfy the functional equation (69) for all $p, q, r, s \in H$. Then either $g, f$ are of the form

$$
\begin{aligned}
& g(p, q)=\frac{1}{2}\left(m_{1}(p) m_{2}(q)+m_{2}(p) m_{1}(q)\right) \\
& f(p, q)=\alpha_{1} m_{1}(p) m_{2}(q)+\alpha_{2} m_{2}(p) m_{1}(q)
\end{aligned}
$$

for all $p, q \in H$, where $m_{1}, m_{2}: H \rightarrow \mathbb{C}$ are exponential functions such that $m_{1} \neq m_{2}$ and $\alpha_{1}, \alpha_{2} \in \mathbb{C}$, or else

$$
\begin{gathered}
g(p, q)=m^{*}(p \circ q), \quad p, q \in H, \\
f(p, q)= \begin{cases}m^{*}(p \circ q)\left(\beta+a^{*}(p)-a^{*}(q)\right), & \text { if } p, q \in H \backslash I, \\
0, & \text { if } p \in I \text { or } q \in I,\end{cases}
\end{gathered}
$$

where $I=\left\{p \in H \mid m^{*}(p)=0\right\}, a^{*}: H \backslash I \rightarrow \mathbb{C}$ is an additive function, $m^{*}: H \rightarrow \mathbb{C}$ is an exponential function, and $\beta \in \mathbb{C}$.

Proof. Let $\sigma(p, q)=(q, p)$ for all $(p, q) \in S$. Then by Theorem 2 for the case when $m \circ \sigma \neq m$, all general solutions $(f, g)$ of $(69)$ are given by

$$
\begin{aligned}
& g(p, q)=\frac{m(p, q)+m(q, p)}{2}, \\
& f(p, q)=\alpha_{1} m(p, q)+\alpha_{2} m(q, p)
\end{aligned}
$$

for all $p, q \in H$, where $m: S \rightarrow \mathbb{C}$ is an exponential function and $\alpha_{1}, \alpha_{2} \in \mathbb{C}$. Since $m$ can be written in the form $m(p, q)=$ $m_{1}(p) m_{2}(q)$, for some exponential functions $m_{1}, m_{2}: H \rightarrow$ $\mathbb{C}$, from (73) we get (71). Assume that $m=m \circ \sigma$; that is, $m(p, q)=m(q, p)$, for all $p, q \in H$. Then we have

$$
m(p, q)=m_{1}(p) m_{2}(q)=m_{1}(q) m_{2}(p)
$$

for all $p, q \in H$. Let $I_{1}=\left\{p \in H \mid m_{1}(p)=0\right\}, I_{2}=\{p \in H \mid$ $\left.m_{2}(p)=0\right\}$. If there exists $q_{0} \in I_{1} \backslash I_{2}$, putting $q=q_{0}$ in (74) we have $m_{1}(p)=0$, for all $\in H$, and hence $m=g=0$. Since we exclude the case $f=0$ or $g=0$, we have $I_{1} \subset I_{2}$. Similarly, we have $I_{2} \subset I_{1}$ and $I_{1}=I_{2}:=I$. From (74), we have

$$
\frac{m_{1}(p)}{m_{2}(p)}=\frac{m_{1}(q)}{m_{2}(q)}
$$

for all $p, q \notin I$, which implies

$$
m_{1}(p)=\alpha m_{2}(p)
$$

for all $p \notin I$ and for some $\alpha \in \mathbb{C}$. Thus, we have

$$
m_{1}(p)^{2}=\alpha^{2} m_{2}(p)^{2}=\alpha \cdot \alpha m_{2}\left(p^{2}\right)=\alpha m_{1}\left(p^{2}\right)=\alpha m_{1}(p)^{2}
$$

for all $p \notin I$, which implies $\alpha=1$ and hence $m_{1}=m_{2}:=m^{*}$. Thus, from (73) and (74) we have

$$
\begin{aligned}
g(p, q) & =\frac{m(p, q)+m(q, p)}{2}=m(p, q) \\
& =m_{1}(p) m_{2}(q)=m^{*}(p \circ q)
\end{aligned}
$$

for all $p, q \in H$. Since $m(p, q)=0$ if and only if $p \in I$ or $q \in I$, by Remark 3 we have

$$
\begin{aligned}
f(p, q) & =m^{*}(p \circ q)(\beta+a(p, q)-a(q, p)) \\
& =m^{*}(p \circ q)\left(\beta+a_{1}(p)+a_{2}(q)-a_{1}(q)-a_{2}(p)\right) \\
& =m^{*}(p \circ q)\left(\beta+\left(a_{1}-a_{2}\right)(p)-\left(a_{1}-a_{2}\right)(q)\right) \\
& :=m^{*}(p \circ q)\left(\beta+a^{*}(p)-a^{*}(q)\right)
\end{aligned}
$$

for all $p, q \in H \backslash I$, where $a^{*}: H \backslash I \rightarrow \mathbb{C}$ is an additive function and $\beta \in \mathbb{C}$. Thus, we get (72). This completes the proof.

Remark 9. Since $H=(0,1]$ has the property stated in Remark 3, we have $I=\left\{p \in H \mid m^{*}(p)=0\right\}=\emptyset$. Thus, Theorem 8 includes the result of Riedel and Sahoo [22].

Using Theorem 7, we have the following.

Theorem 10. Let $f, g: S \rightarrow \mathbb{C}$ satisfy the functional equation (70) for all $p, q, r, s \in H$. Then $g, f$ are of the form

$$
\begin{aligned}
& g(p, q)=\frac{m_{1}(p) m_{2}(q)+m_{2}(p) m_{1}(q)}{2}, \\
& f(p, q)=\frac{m_{1}(p) m_{2}(q)+m_{2}(p) m_{1}(q)}{2 \beta}
\end{aligned}
$$

for all $p, q \in H$, where $m_{1}, m_{2}: H \rightarrow \mathbb{C}$ are exponential functions and $\beta \in \mathbb{C}$ with $\beta \neq 0$.

Using polar form of complex numbers, we have the following two lemmas. In the following we define $\arg (z)=0$ for $z=0$.

Lemma 11. Let $m: \mathbb{C} \rightarrow \mathbb{C}$ satisfy the functional equation

$$
m(z w)=m(z) m(w)
$$

for all $z, w \in \mathbb{C}$. Then $m$ has the form

$$
m(z)=M(|z|) E(\arg z)
$$

for all $z \in \mathbb{C}$, where $M: \mathbb{R} \rightarrow \mathbb{C}$ is a multiplicative function, that is, $M(x y)=M(x) M(y)$, for all $x, y \in \mathbb{R}$, and $E: \mathbb{R} \rightarrow \mathbb{C}$ is an exponential function such that $E(x+2 \pi)=E(x)$ for all $x \in \mathbb{R}$.

Lemma 12. Let $a: \mathbb{C} \rightarrow \mathbb{C}$ satisfy the functional equation

$$
a(z w)=a(z)+a(w)
$$

for all $z, w \in \mathbb{C}$. Then a has the form

$$
a(z)=M(|z|)+A(\arg z)
$$

for all $z \in \mathbb{C}$, where $M: \mathbb{R} \rightarrow \mathbb{C}$ is a multiplicative function and $A: \mathbb{R} \rightarrow \mathbb{C}$ is an additive function such that $A(x+2 \pi)=$ $A(x)$ for all $x \in \mathbb{R}$.

Using Theorem 2 and Lemma 11 we have the following. 
Theorem 13. Let $f, g: \mathbb{C} \rightarrow \mathbb{C}$ satisfy the functional equation

$$
f(z w)+f(z \bar{w})=2 f(z) g(w)
$$

for all $z, w \in \mathbb{C}$. Then $f, g$ are of the form

$$
\begin{aligned}
& f(z)=M(|z|)\left(\alpha_{1} E(\arg z)+\alpha_{2} E(\arg \bar{z})\right), \\
& g(z)=\frac{1}{2} M(|z|)(E(\arg z)+E(\arg \bar{z}))
\end{aligned}
$$

for all $z \in \mathbb{C}$, or

$$
\begin{aligned}
& g(z)=M(|z|), \\
& f(z)=M(|z|)(\beta+A(\arg z))
\end{aligned}
$$

for all $z \in \mathbb{C}$, where $M: \mathbb{R} \rightarrow \mathbb{C}$ is a multiplicative function, $E: \mathbb{R} \rightarrow \mathbb{C}$ is an exponential function such that $E(x+2 \pi)=$ $E(x)$, for all $x \in \mathbb{R}$, and $A: \mathbb{R} \rightarrow \mathbb{C}$ is an additive function such that $A(x+2 \pi)=A(x)$, for all $x \in \mathbb{R}$, and $\alpha_{1}, \alpha_{2}, \beta \in \mathbb{C}$.

Remark 14. If some regularities on $m$ are assumed in Theorem 13, it can be verified that $E=1$ and $A=0$. Thus, all regular solutions $(g, f)$ (e.g., continuous solutions) of $(85)$ are given by

$$
g(z)=|z|^{c}, \quad f(z)=\beta|z|^{c}
$$

for all $z \in \mathbb{C}$, where $c, \beta \in \mathbb{C}$.

Using Theorem 7 and Lemma 11 we have the following.

Theorem 15. Let $f, g: \mathbb{C} \rightarrow \mathbb{C}$ satisfy the functional equation

$$
f(z w)+f(z \bar{w})=2 g(z) f(w)
$$

for all $z, w \in \mathbb{C}$. Then $f, g$ are of the form

$$
\begin{aligned}
& f(z)=\alpha M(|z|)(E(\arg z)+E(\arg \bar{z})), \\
& g(z)=\frac{1}{2} M(|z|)(E(\arg z)+E(\arg \bar{z}))
\end{aligned}
$$

for all $z \in \mathbb{C}$, where $M: \mathbb{R} \rightarrow \mathbb{C}$ is a multiplicative function and $\alpha \in \mathbb{C}$.

As consequence of Theorems 13 and 15, we also find general solutions of the following functional equations:

$$
\begin{aligned}
& f(u x-v y, u y+v x)+f(u x+v y, u y-v x) \\
&-2 f(x, y) g(u, v)=0, \\
& f(u x-v y, u y+v x)+f(u x+v y, u y-v x) \\
&-2 g(x, y) f(u, v)=0, \\
& f(u x+v y, u y+v x)+f(u x-v y, u y-v x) \\
& \quad-2 f(x, y) g(u, v)=0, \\
& f(u x+v y, u y+v x)+f(u x-v y, u y-v x) \\
&-2 g(x, y) f(u, v)=0,
\end{aligned}
$$

for all $x, y, u, v \in \mathbb{R}$, where $f, g: \mathbb{R}^{2} \rightarrow \mathbb{R}$.
Theorem 16. Let $f, g: \mathbb{R}^{2} \rightarrow \mathbb{C}$ satisfy the functional equation (91). Then $f, g$ are of the form

$$
\begin{aligned}
f(x, y)= & M\left(\sqrt{x^{2}+y^{2}}\right) \\
& \times\left(\alpha_{1} E\left(\arctan \left(\frac{y}{x}\right)\right)+\alpha_{2} E\left(-\arctan \left(\frac{y}{x}\right)\right)\right), \\
g(x, y)= & \frac{1}{2} M\left(\sqrt{x^{2}+y^{2}}\right) \\
& \times\left(E\left(\arctan \left(\frac{y}{x}\right)\right)+E\left(-\arctan \left(\frac{y}{x}\right)\right)\right)
\end{aligned}
$$

for all $(x, y) \in \mathbb{R}^{2}$, or

$$
\begin{aligned}
& g(x, y)=M\left(\sqrt{x^{2}+y^{2}}\right), \\
& f(x, y)=M\left(\sqrt{x^{2}+y^{2}}\right)\left(\beta+A\left(\arctan \left(\frac{y}{x}\right)\right)\right)
\end{aligned}
$$

for all $(x, y) \in \mathbb{R}^{2}$, where $M:[0, \infty) \rightarrow \mathbb{C}$ is a multiplicative function, $E: \mathbb{R} \rightarrow \mathbb{C}$ is an exponential function such that $E(x+2 \pi)=E(x)$, for all $x \in \mathbb{R}$, and $A: \mathbb{R} \rightarrow \mathbb{C}$ is an additive function such that $A(x+2 \pi)=A(x)$, for all $x \in \mathbb{R}$, and $\alpha_{1}, \alpha_{2}, \beta \in \mathbb{C}$.

Proof. Define $F, G: \mathbb{C} \rightarrow \mathbb{R}$ by

$$
F(x+i y)=f(x, y), \quad G(x+i y)=g(x, y)
$$

for all $x, y \in \mathbb{R}$. Then, (91) is reduced to

$$
F(z w)+F(z \bar{w})-2 F(z) G(w)=0
$$

for all $z, w \in \mathbb{C}$. Using Theorem 13 we get the result.

Similarly, using Theorem 15 we have the following.

Theorem 17. Let $f, g: \mathbb{R}^{2} \rightarrow \mathbb{C}$ satisfy the functional equation (92). Then $f, g$ are of the form

$$
\begin{aligned}
f(x, y)= & \alpha M\left(\sqrt{x^{2}+y^{2}}\right) \\
& \times\left(E\left(\arctan \left(\frac{y}{x}\right)\right)+E\left(-\arctan \left(\frac{y}{x}\right)\right)\right), \\
g(x, y)= & \frac{1}{2} M\left(\sqrt{x^{2}+y^{2}}\right) \\
& \times\left(E\left(\arctan \left(\frac{y}{x}\right)\right)+E\left(-\arctan \left(\frac{y}{x}\right)\right)\right),
\end{aligned}
$$

for all $(x, y) \in \mathbb{R}^{2}$, where $M:[0, \infty) \rightarrow \mathbb{C}$ is a multiplicative function, $E: \mathbb{R} \rightarrow \mathbb{C}$ is an exponential function such that $E(x+2 \pi)=E(x)$, for all $x \in \mathbb{R}$, and $\alpha \in \mathbb{C}$. 
Theorem 18. Let $f, g: \mathbb{R}^{2} \rightarrow \mathbb{C}$ satisfy the functional equation (93). Then either $g$, $f$ are of the form

$$
\begin{aligned}
g(p, q)= & \frac{1}{2}\left(M_{1}(p+q) M_{2}(p-q)\right. \\
& \left.+M_{2}(p+q) M_{1}(p-q)\right) \\
f(p, q)= & \alpha_{1} M_{1}(p+q) M_{2}(p-q) \\
& +\alpha_{2} M_{2}(p+q) M_{1}(p-q)
\end{aligned}
$$

for all $p, q \in \mathbb{R}$, where $M_{1}, M_{2}: \mathbb{R} \rightarrow \mathbb{C}$ are multiplicative functions such that $M_{1} \neq M_{2}$ and $\alpha_{1}, \alpha_{2} \in \mathbb{C}$, or

$$
\begin{gathered}
g(p, q)=M\left(p^{2}-q^{2}\right), \quad p, q \in \mathbb{R}, \\
f(p, q)= \begin{cases}M\left(p^{2}-q^{2}\right)(\beta+L(p+q)-L(p-q)), & \text { if } p^{2} \neq q^{2}, \\
0, & \text { if } p^{2}=q^{2},\end{cases}
\end{gathered}
$$

where $L: \mathbb{R} \backslash\{0\} \rightarrow \mathbb{C}$ is a logarithmic function, that is, $L(x y)=L(x)+L(y), M: \mathbb{R} \rightarrow \mathbb{C}$ is a multiplicative function, and $\beta \in \mathbb{C}$, or

$$
g(p, q)=1, \quad f(p, q)=\beta
$$

for all $p, q \in \mathbb{R}$.

Proof. Define $F, G: \mathbb{R}^{2} \rightarrow \mathbb{R}$ by

$$
\begin{aligned}
& F(x, y)=f\left(\frac{x+y}{2}, \frac{x-y}{2}\right), \\
& G(x, y)=g\left(\frac{x+y}{2}, \frac{x-y}{2}\right)
\end{aligned}
$$

for all $x, y \in \mathbb{R}$. Then, (93) is reduced to

$$
F(p r, q s)+F(p s, q r)=2 F(p, q) G(r, s)
$$

for all $p, q, r, s \in \mathbb{R}$. Applying Theorem 8 with $H=\langle\mathbb{R}, \times\rangle$ for $m \neq m \sigma$, we get (100). It is easy to see that $I:=\{x \in$ $\left.\mathbb{R}: m^{*}(x)=0\right\}=\{0\}$ or $I=\emptyset$ in the case when $m=m \sigma$ of Theorem 8. If $I=\{0\}$, from (71) we get (101) with $M=$ $m^{*}, L=a^{*}$. If $I=\emptyset$, then $m^{*}(0) \neq 0$ and it follows that $g=m^{*}=1$. In this case, $a^{*}$ is a logarithmic function on $\mathbb{R}$; that is, $a^{*}$ satisfies

$$
a^{*}(p q)=a^{*}(p)+a^{*}(q)
$$

for all $p, q \in \mathbb{R}$. Putting $q=0$ in (105) we have $a^{*}=0$. Thus, from (71) we get (102). This completes the proof.

Define $F, G: \mathbb{R}^{2} \rightarrow \mathbb{R}$ by

$$
\begin{aligned}
& F(x, y)=f\left(\frac{x+y}{2}, \frac{x-y}{2}\right), \\
& G(x, y)=g\left(\frac{x+y}{2}, \frac{x-y}{2}\right)
\end{aligned}
$$

for all $x, y \in \mathbb{R}$. Then, (94) is reduced to

$$
F(p r, q s)+F(p s, q r)=2 G(p, q) F(r, s)
$$

for all $p, q, r, s \in \mathbb{R}$. Using Theorem 10 we have the following.
Theorem 19. Let $f, g: \mathbb{R}^{2} \rightarrow \mathbb{C}$ satisfy the functional equation (94). Then either $g$ or $f$ has the form

$$
\begin{aligned}
& g(p, q)=\frac{M_{1}(p+q) M_{2}(p-q)+M_{2}(p+q) M_{1}(p-q)}{2}, \\
& f(p, q)=\frac{M_{1}(p+q) M_{2}(p-q)+M_{2}(p+q) M_{1}(p-q)}{2 \beta},
\end{aligned}
$$

for all $p, q \in \mathbb{R}$, where $M_{1}, M_{2}: \mathbb{R} \rightarrow \mathbb{C}$ are multiplicative functions and $\beta \in \mathbb{C}$ with $\beta \neq 0$.

\section{Conflict of Interests}

The authors declare that there is no conflict of interests regarding the publication of this paper.

\section{Acknowledgments}

The authors are very thankful to the referee for valuable suggestions that improved the presentation of the paper. This work was done while the first author visited the University of Louisville from Kunsan National University during 2012-13. This work was supported by Basic Science Research Program through the National Research Foundation of Korea (NRF) funded by the Ministry of Education, Science and Technology (MEST) (no. 2012008507).

\section{References}

[1] J. d'Alembert, "Addition au Mémoire sur la courbe que forme une corde tendue mise en vibration," Histoire de l'Académie Royale, pp. 355-360, 1750.

[2] P. K. Sahoo and Pl. Kannappan, Introduction to Functional Equations, CRC Press, Boca Raton, Fla, USA, 2011.

[3] H. Stetkær, Functional Equations on Groups, World Scientific, Singapore, 2013.

[4] P. Sinopoulos, "Functional equations on semigroups," Aequationes Mathematicae, vol. 59, no. 3, pp. 255-261, 2000.

[5] W. H. Wilson, "On certain related functional equations," Bulletin of the American Mathematical Society, vol. 26, no. 7, pp. 300-312, 1920.

[6] W. H. Wilson, "Two general functional equations," Bulletin of the American Mathematical Society, vol. 31, no. 7, pp. 330-334, 1925.

[7] S. Kaczmarz, "Sur l'équation fonctionnelle $f(x)+f(x+y)=$ $\phi(y) f(x+y / 2)$," Fundamenta Mathematicae, vol. 6, pp. 122129, 1924.

[8] G. van der Lyn, "Sur l'équation fonctionnelle $f(x+y)+f(x-$ $y)=2 f(x) \emptyset(y)$," Mathematica, vol. 16, pp. 91-96, 1940.

[9] I. Fenyö, "Uber eineLösungsmethode gewisser Funktionalgleichungen," Acta Mathematica Academiae Scientiarum Hungaricae, vol. 7, pp. 383-396, 1956.

[10] J. K. Chung, B. R. Ebanks, C. T. Ng, and P. K. Sahoo, "On a quadratic-trigonometric functional equation and some applications," Transactions of the American Mathematical Society, vol. 347, no. 4, pp. 1131-1161, 1995.

[11] J. Aczél, Lectures on Functional Equations and Their Applications, Dover Publications, New York, NY, USA, 2006. 
[12] H. Stetkær, “D’Alembert's and Wilson's functional equations for vector and $2 \times 2$ matrix valued functions," Mathematica Scandinavica, vol. 87, no. 1, pp. 115-132, 2000.

[13] J. Aczél and J. Dhombres, Functional Equations in Several Variables, Cambridge University Press, New York, NY, USA, 1989.

[14] A. Bahyrycz, "On solutions of the second generalization of d'Alembert's functional equation on a restricted domain," Applied Mathematics and Computation, vol. 223, pp. 209-215, 2013.

[15] I. Corovei, "The functional equation $f(x y)+f\left(x y^{-1}\right)=$ $2 f(x) g(y)$ for nilpotent groups," Mathematica, vol. 22, no. 1, pp. 33-41, 1980.

[16] I. Corovei, "Wilson's functional equation on $P_{3}$-groups," Aequationes Mathematicae, vol. 61, no. 3, pp. 212-220, 2001.

[17] I. Corovei, "Wilson's functional equation on metabelian groups," Mathematica, vol. 44, no. 2, pp. 137-146, 2002.

[18] P. de Place Friis, "d'Alembert's and Wilson's equations on Lie groups," Aequationes Mathematicae, vol. 67, no. 1-2, pp. 12-25, 2004.

[19] Z. Fechner, "Wilson's functional equation in Banach algebras," Acta Scientiarum Mathematicarum, vol. 75, no. 1-2, pp. 131-142, 2009.

[20] Z. Fechner, "Wilson's functional equation in algebras," Studia Scientiarum Mathematicarum Hungarica, vol. 47, no. 3, pp. 388400, 2010.

[21] H. Stetkær, "Functional equations on abelian groups with involution," Aequationes Mathematicae, vol. 54, no. 1-2, pp. 144$172,1997$.

[22] T. Riedel and P. K. Sahoo, "On a generalization of a functional equation associated with the distance between the probability distributions," Publicationes Mathematicae Debrecen, vol. 46, no. 1-2, pp. 125-135, 1995. 


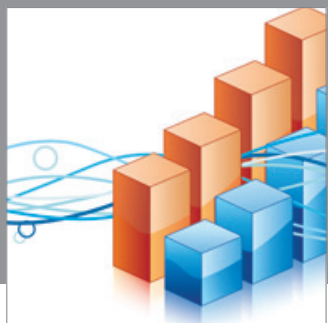

Advances in

Operations Research

mansans

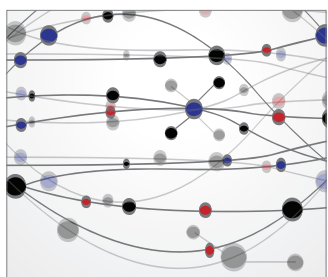

The Scientific World Journal
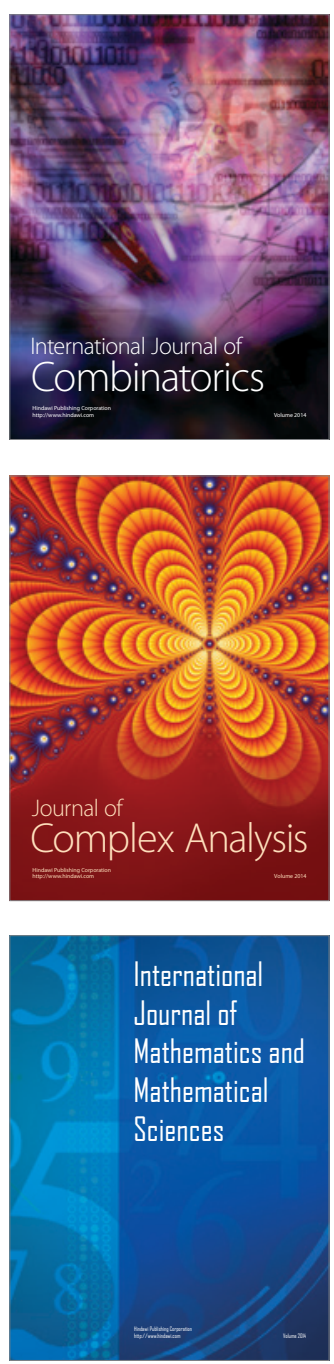
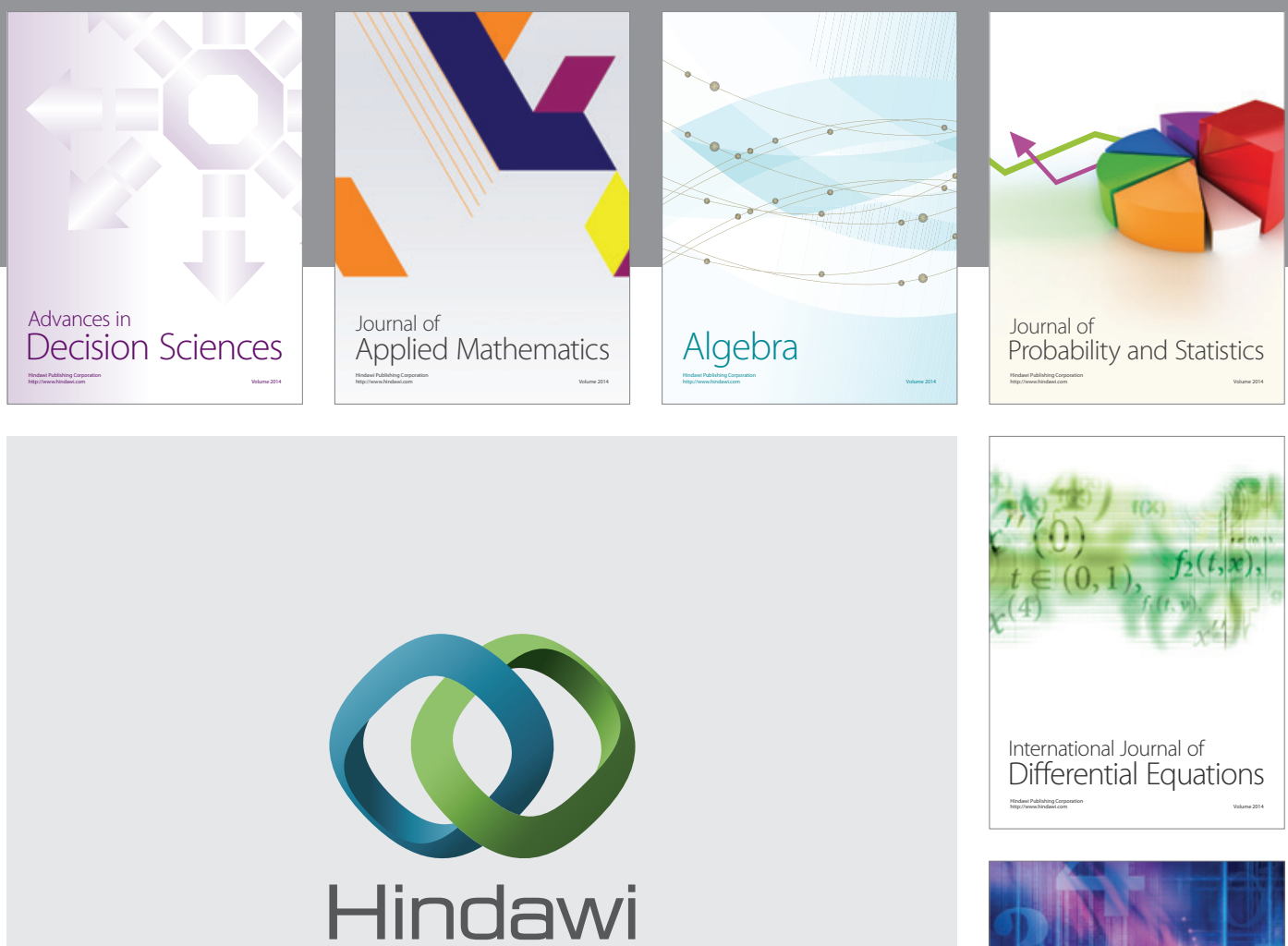

Submit your manuscripts at http://www.hindawi.com
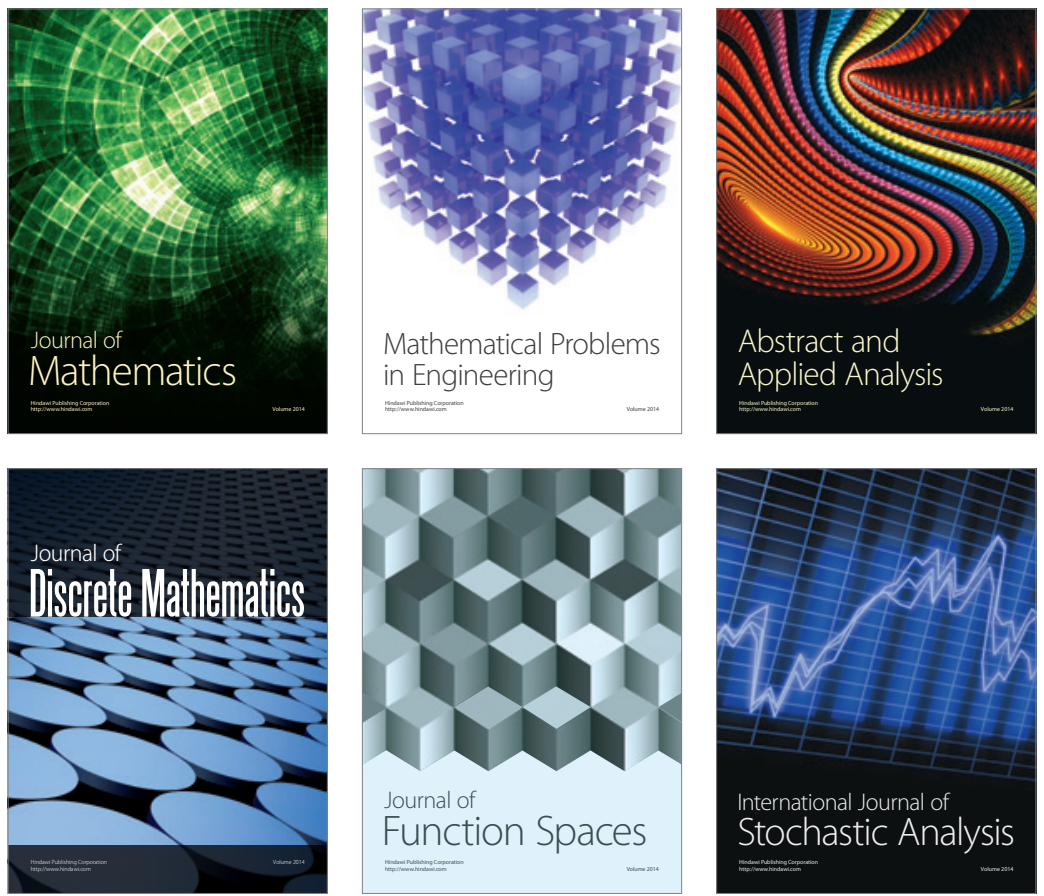

Journal of

Function Spaces

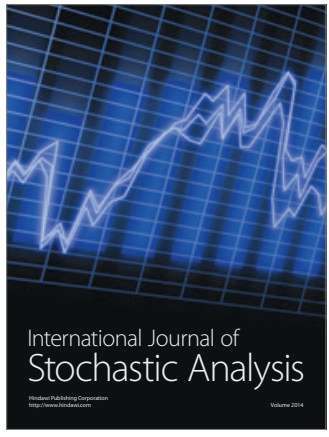

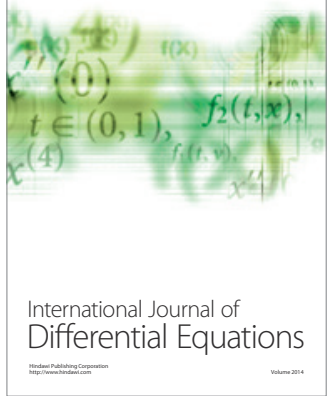
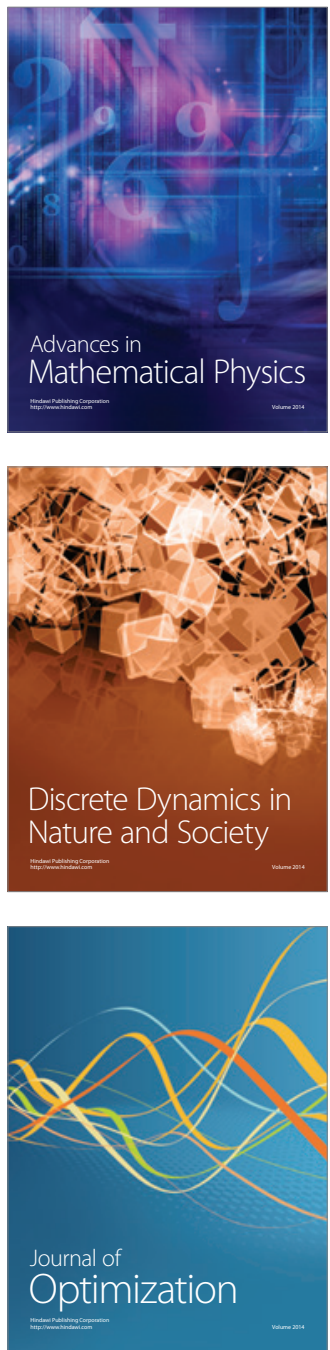